\title{
Astrocitomas pilomixoides. Presentación de tres casos y revisión de la literatura
}

\author{
M. Domínguez-Páez; B. Weil-Lara*; S. Rodríguez-Barceló; J.M. Medina-Imbroda; M. Puch-Ramírez; B. Ros-López \\ y M.A. Arráez-Sánchez \\ Servicio de Neurocirugía, Sección de Neurocirugía Infantil. *Servicio de Anatomía Patológica. Hospital Regional Universitario Carlos Haya, \\ Málaga.
}

\section{Resumen}

Introducción. El astrocitoma pilomixoide (APM) es un tumor del sistema nervioso central (SNC) con características clínicas y anatomopatológicas propias que lo convierten en una entidad diferente del astrocitoma pilocítico (AP). Este tumor aparece en la clasificación de tumores del SNC de la OMS en su cuarta edición (año 2007) como subtipo de AP dentro del grupo de los tumores de origen astrocitario. Actualmente sigue siendo una entidad tumoral poco conocida, existiendo controversia sobre su origen histológico y su comportamiento clínico, y una falta de consenso en cuanto a su manejo terapéutico.

Objetivo. Revisar la literatura científica relacionada con el tema y presentar tres casos valorados en nuestro servicio.

Conclusiones. El APM es una entidad anatomopatológica relacionada con el AP aunque con una mayor tendencia a la recidiva $\mathrm{y}$ diseminación por el líquido cefalorraquídeo, por lo que se recomienda seguimiento estricto y tratamiento oncológico adyuvante.

PALABRAS CLAVE: Astrocitoma pilocítico. Astrocitoma pilomixoide.

Pilomyxoid astrocytoma. Three cases and review

\section{Summary}

Introduction. Pilomyxoid astrocytoma (PMA) is a central nervous system (CNS) tumour with peculiar clinicopathological features, that turn it into an entity different from pilocytic astrocytoma (PA). It appears in 2007 WHO classification of tumours of the CNS as an PA subtype belonging to the group of astrocytic tumours. Nowadays little is still known about this tumour entity; the histological origin and clinical beha-

Recibido: 28-09-08. Aceptado: 25-04-09 vior remain controversial, and there is no consensus about its management.

Objective. To review the scientific literature related to the topic and to present three cases treated at our service.

Conclusions. PMA is an histological entity related to PA with a greater trend to regrowth and cerebrospinal fluid dissemination, therefore strict follow-up and oncological treatment is recommended.

KEY WORDS: Pilocytic astrocytoma. Pilomyxoid astrocytoma.

\section{Introducción}

En 1985 Jänisch describió el "astrocitoma pilocítico diencefálico de aparición en la infancia" como una tumoración con características histológicas peculiares tales como la ausencia de fibras de Roshental, sin llegar a considerarlo una entidad diferente del astrocitoma pilocítico (AP) típico ${ }^{11}$. No es hasta 1999 cuando Tihan introdujo el término "astrocitoma pilomixoide" (APM) para referirse a estas lesiones como entidad clínico-patológica propia, señalando una mayor tendencia a la recidiva y a la diseminación por el líquido cefalorraquídeo (LCR), y por lo tanto un peor pronóstico vital ${ }^{23}$. Este comportamiento más agresivo ha sido destacado también por otros autores ${ }^{14}$. Recientemente el APM aparece en la clasificación de tumores del Sistema Nervioso Central (SNC) de la OMS en su cuarta edición (año 2007) como subtipo de AP dentro del grupo de los tumores de origen astrocitario, considerándose

Abreviaturas. AME: antígeno de membrana epitelial. AP: astrocitoma pilocítico. APM: astrocitoma pilomixoide. Cr Phy: cromosoma Philadelphia. CNS: central nervous system. CSF: cerebral spinal fluid. HTIC: hipertensión intracraneal. LCR: líquido cefalorraquídeo. LGG: low grade glioma. LMC: leucemia mieloide crónica. OMS: organización mundial de la salud. PA: pilocytic astrocytoma. PMA: pilomyxod astrocytoma. RMN: resonancia magnética nuclear. SIOP: society of pediatric oncology. SNC: sitema nervioso central. WHO: world health organization. 


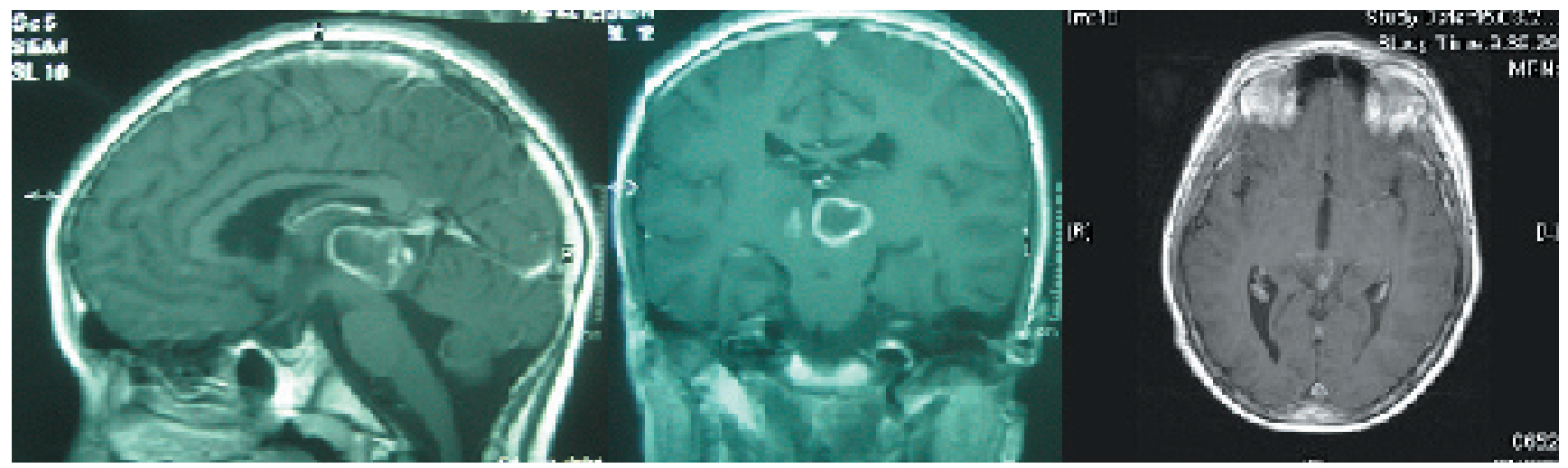

Caso 1. Imagen $1 a$ (izquierda): sagital T1 gadolinio. Imagen 1 (centro): coronal T1 gadolinio. Imagen 1c (derecha): axial T1 gadolinio postradioterapia.

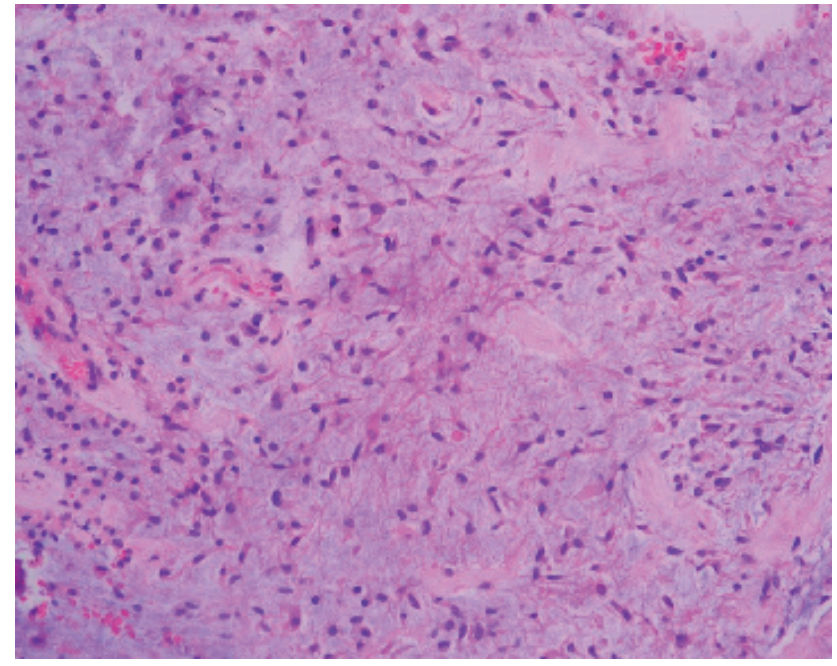

Caso 1. Imagen 1.1. (Hematoxilina-Eosina 20x) Matriz mixoide y células piloides de núcleos hipercromáticos y prolongaciones citoplasmáticas eosinófilas.

un astrocitoma grado $\mathrm{II}^{22}$. El APM podría ser una forma inmadura de $\mathrm{AP}^{3,5,8}$ con preferencia por la localización hipotálamo-quiasmática ${ }^{5,18}$. Actualmente continúa siendo una entidad tumoral poco conocida, existiendo controversia sobre su origen histológico y una falta de consenso en cuanto a su manejo terapéutico. Presentamos tres casos de reciente diagnóstico y tratamiento en nuestro servicio, junto una revisión bibliográfica de dicha entidad tumoral.

\section{Caso 1}

Niño de 11 años de edad que debutó con cuadro de cefalea, vómitos, alteración de la marcha y un signo de Parinaud en la exploración neurológica. En la resonancia magnética (RM) craneal practicada se observó una tumoración a nivel de la porción posterior del tercer ventrículo de aproximadamente $3 \times 2 \mathrm{~cm}$. de diámetro, de centro necrótico-quístico

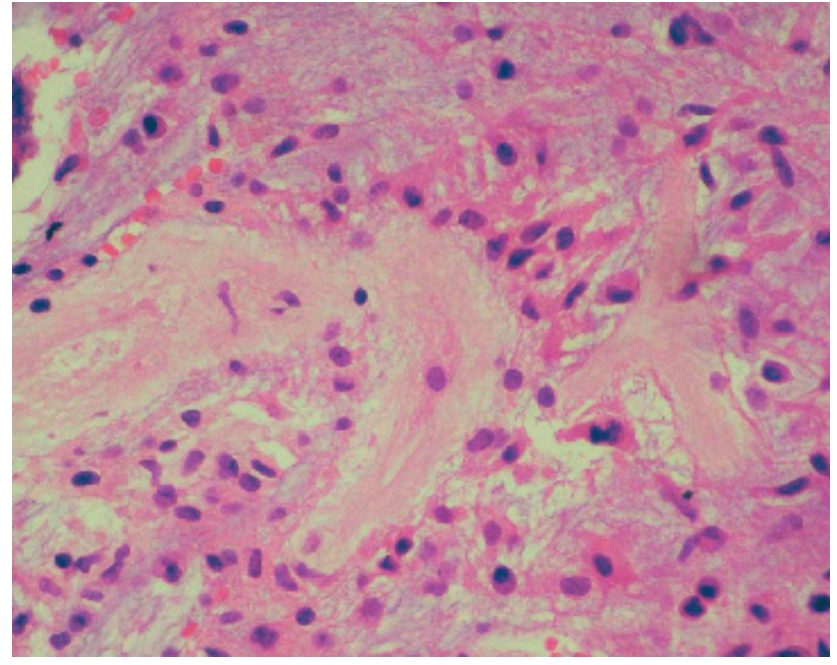

Caso 1. Imagen 1.2. (Hematoxilina-Eosina 40x) Las células tienden a agruparse en torno a vasos.

y captación periférica de contraste, y que se asociaba a una hidrocefalia obstructiva (ver imagen 1a y 1b). Fue intervenido quirúrgicamente mediante abordaje endoscópico con biopsia y ventriculostomía premamilar, realizándose posteriormente una derivación ventrículo-peritoneal por ventriculostomía no funcionante. La biopsia evidenció una proliferación de células bipolares con características piloides, de núcleos hipercromáticos y prolongaciones citoplasmáticas eosinofílicas inmersas en abundante estroma mixoide y con tendencia a disponerse en torno a los vasos. Se observó necrosis y aisladas figuras de mitosis, sin embargo no se observaron fibras de Rosenthal ni cuerpos granulares. Las células fueron positivas para la proteína glial fibrilar ácida (PGFA. Ver imágenes 1.1 y 1.2). El diagnóstico histológico fue de APM. El caso se valoró en comité oncológico decidiéndose, dada la edad del paciente y los hallazgos en la exploración, tratamiento radioterápico. 


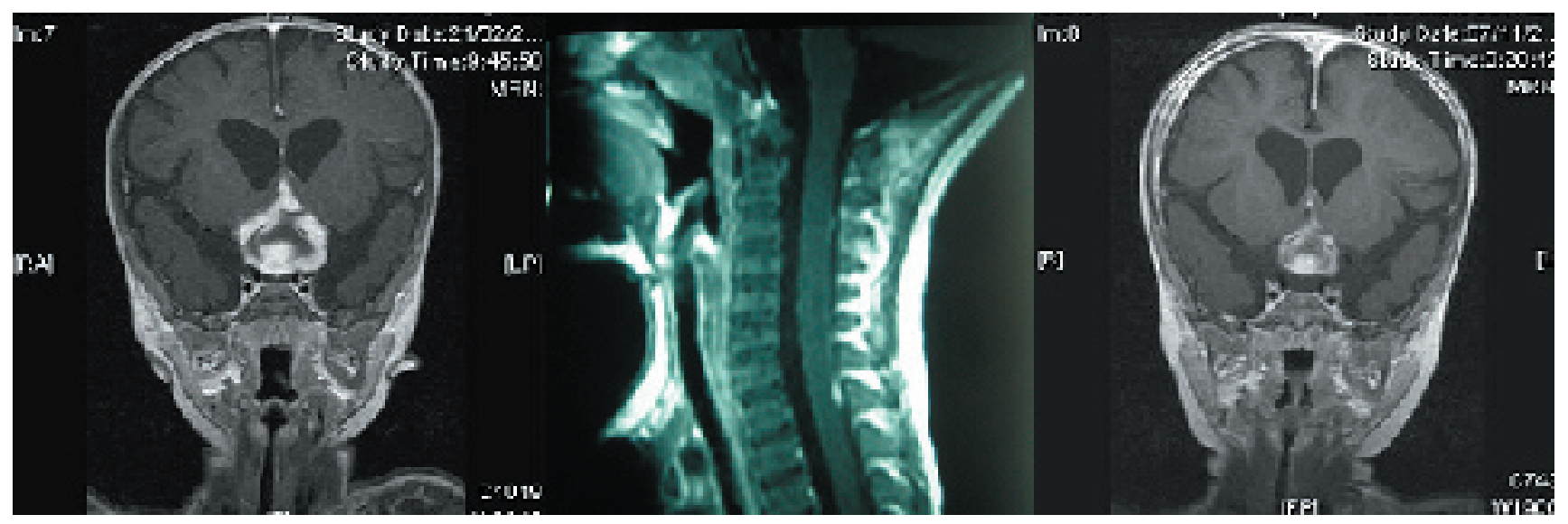

Caso 2. Imagen $2 a$ (izquierda): coronal T1 gadolinio. Imagen $2 b$ (centro): diseminación leptomeningea cervical. Imagen $2 c$ (derecha): reducción de tamaño tumoral tras quimioterapia.

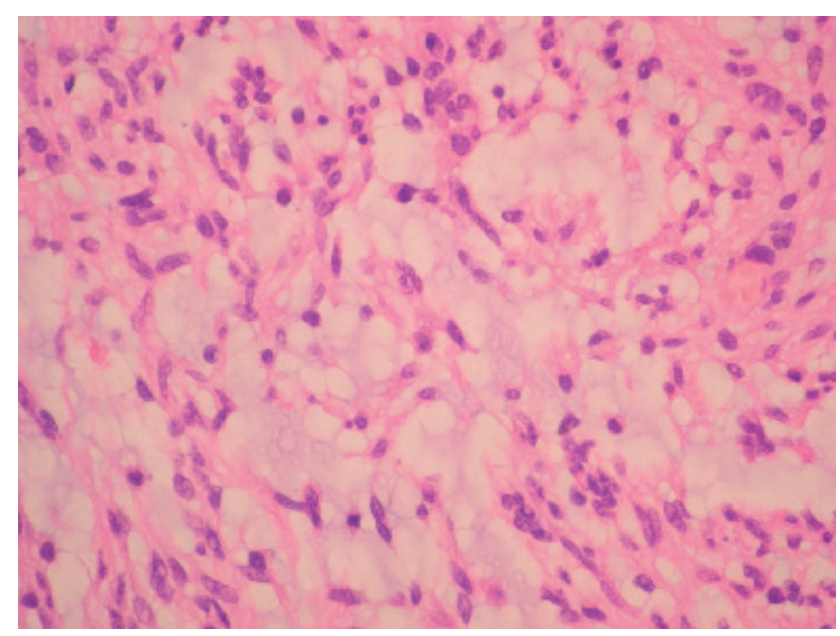

Caso 2. Imagen 2.1. (Hematoxilina-Eosina 40x) Células bipolares con prolongaciones eosinófilas inmersas en estroma mixoide.

El paciente, 36 meses después, se encuentra asintomático, persistiendo el signo de Parinaud. La RM muestra una pequeña lesión residual (ver imagen 1c).

\section{Caso 2}

Niño de seis meses de edad remitido a nuestro servicio por macrocefalia progresiva y nistagmus. Se le practicó una RM craneal en la que se objetivó una tumoración a nivel hipotálamo-quiasmático de aproximadamente $4 \times 3 \mathrm{~cm}$. de diámetro con hidrocefalia obstructiva (ver imagen 2a), y RM espinal donde se observaron dos lesiones hipercaptantes en la porción dorsal del cordón medular cervical, compatibles con diseminación del tumor por el LCR (ver imagen 2b). Fue intervenido quirúrgicamente mediante biopsia endoscópica de la lesión y derivación ventrículo-peritoneal. La biopsia evidenció una proliferación de células bipolares con carac-

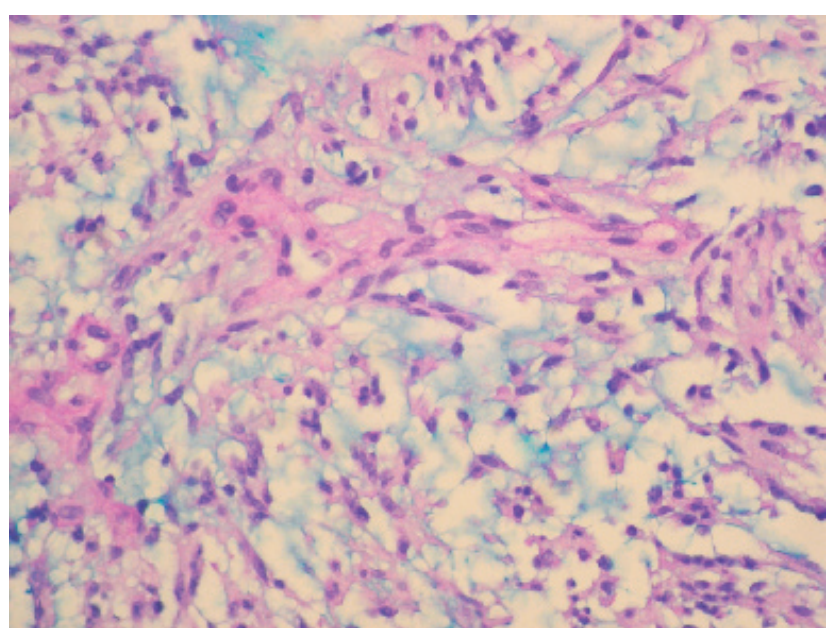

Caso 2. Imagen 2.2. (Azul Alcian-Pas 40X). Abundante estroma mixoide evidenciado con histoquímica.

terísticas piloides, de núcleos hipercromáticos y prolongaciones citoplasmáticas eosinofílicas de disposición errática, que se encontraban inmersas en abundante estroma mixoide evidente con hematoxilina-eosina y técnicas de histoquímica (azul alcian-PAS). No se observaron fibras de Rosenthal ni cuerpos granulares. Las células mostraron positividad para la PGFA y sinaptofisina, siendo negativa la cromogranina, neurofilamentos y el antígeno epitelial de membrana (AME). El Ki67 fue del 2\% (ver imágenes 2.1 y 2.2). El diagnóstico histológico fue de APM. Se decidió en comité oncológico quimioterapia adyuvante con vincristina y carboplatino dada la edad del paciente y la diseminación leptomeníngea (protocolo SIOP-LGG 2004). En la primera RM de control se observó una disminución de tamaño de la lesión diencefálica (ver imagen 2c), habiendo desaparecido las dos lesiones medulares tres meses después del inicio el tratamiento. Tras 18 meses de seguimiento el paciente presenta un retraso psicomotor leve, así como en la adquisición del lenguaje. 


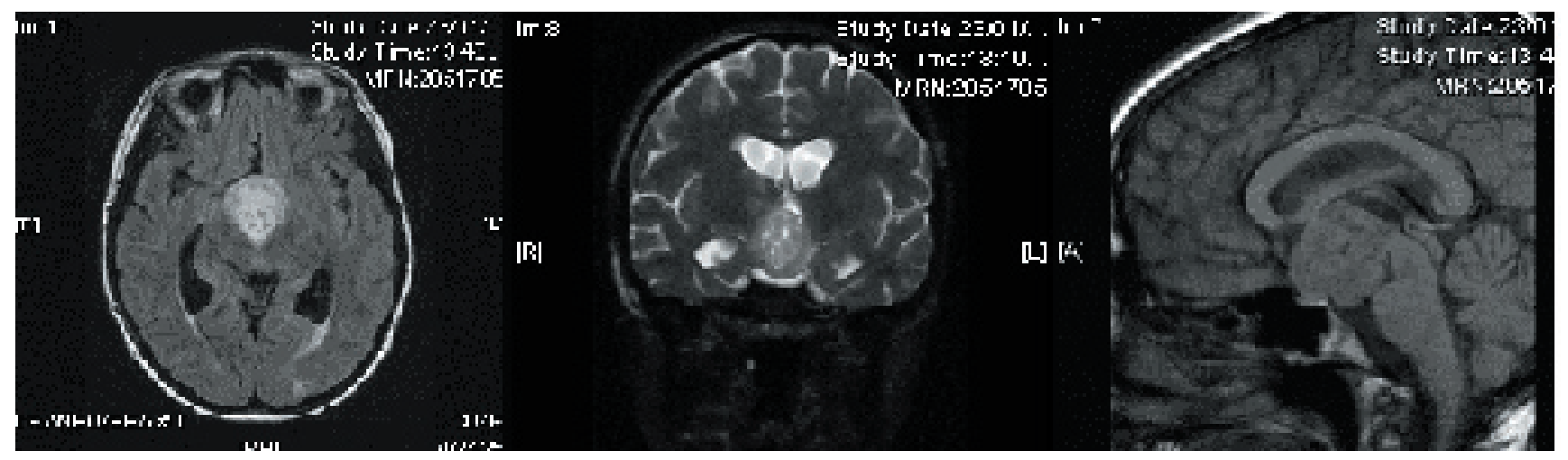

Caso 3. Imagen $3 a$ (izquierda): axial Flair. Imagen 3b (centro): coronal T2. Imagen 3c (derecha): sagital T1.

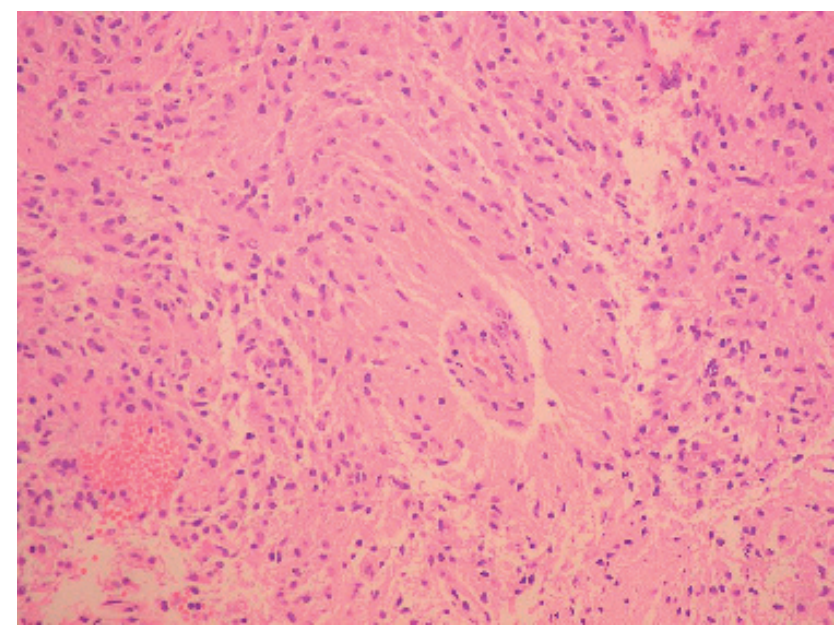

Caso 3. Imagen 3.1 (Hematoxilina-Eosina 20x) Células piloides con disposición angiocéntrica.

\section{Caso 3}

Paciente mujer de 13 años de edad que debutó con cefalea y vómitos. En la RM craneal se apreció una tumoración de III ventrículo con hidrocefalia obstructiva (ver imagen 3a, 3b y 3c). Fue intervenida quirúrgicamente realizándose biopsia endoscópica, septostomía y derivación ventriculoperitoneal. La biopsia evidenció una proliferación de células bipolares con características piloides, con tendencia a la disposición angiocéntrica, inmersas en estroma mixoide. Se observaron focos de necrosis tumoral. No se identificaron fibras de Rosenthal ni cuerpos granulares. Las células mostraron positividad difusa para la PGFA, sinaptofisina y vimentina. El Ki67 fue del 5\%. (ver imágenes 3.1 y 3.2). El diagnóstico histológico fue de APM. En el postoperatorio se detectó déficit hormonal en el eje tiroideo y suprarrenal iniciándose tratamiento sustitutivo. Dada la edad de la paciente y el carácter expansivo de la lesión se propuso tratamiento radioterápico, ya concluido en la actualidad y pendiente de controles de imagen.

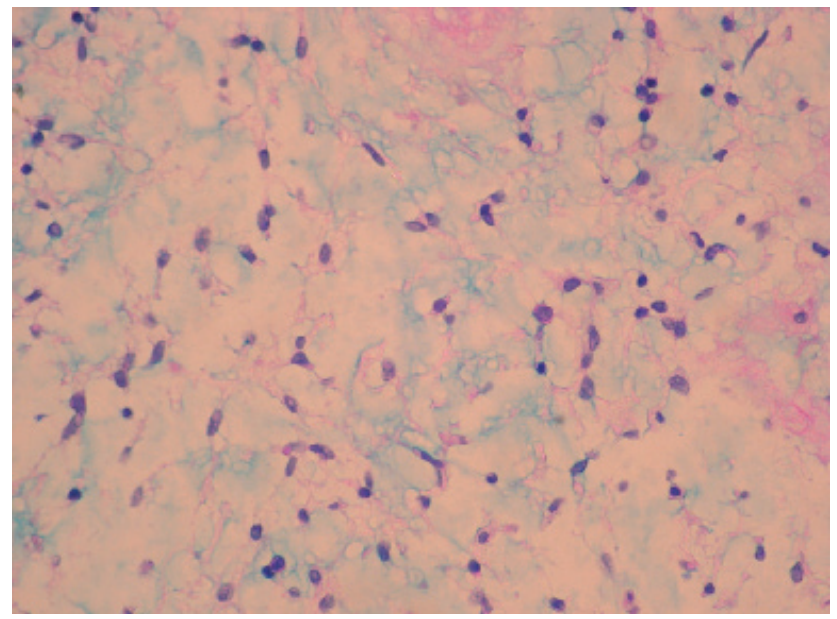

Caso 3. Imagen 3.2 (Azul Alcian-Pas 40X): Abundante estroma mixoide.

\section{Discusión}

El APM fue introducido como entidad clínico-patológica hace aproximadamente 10 años, describiéndose la conducta agresiva de AP diencefálicos en niños en los que se observaron características histológicas peculiares como ausencia de fibras de Rosenthal ${ }^{3,23}$. Posteriormente se describieron series de AP catalogados como atípicos o agresivos que cumplían criterios histológicos de $\mathrm{APM}^{5,8,14}$.

Desde el punto de vista histopatológico se ha propuesto un origen astrocitario del $\mathrm{APM}^{3}$. Esta hipótesis se ha visto apoyada por las semejanzas histológicas descritas entre AP y $\mathrm{APM}^{23}$, así como la existencia de AP con focos de APM $\mathrm{y}_{\text {viceversa }}{ }^{3}$. Se ha descrito un fenómeno de maduración o diferenciación histológica progresiva del APM en AP tras años de seguimiento en recidivas sucesivas del tumor, de modo que el APM podría ser una forma inmadura de $\mathrm{AP}^{3,5,8}$. La presencia de islas gliales con células pilocíticas podrían ser el comienzo de dicho proceso madurativo ${ }^{5}$. Por el contrario se han descrito APM que tras muchos años de evo- 
lución no han sufrido dicho proceso, además de los casos que han aparecido en la edad adulta ${ }^{3}$. El término diferenciación hace referencia a la transformación histológica que sufre el APM secundaria a tratamientos sucesivos con quimioterápicos, sin embargo se han identificado tumores que han sufrido el proceso transformativo y nunca han recibido quimioterapia alguna ${ }^{3,5,8}$. Una segunda hipótesis defiende el origen ependimario del APM basándose en la estructura angiocéntrica de dicho tumor, muy similar a las pseudorrosetas que se han descrito en los ependimomas y tanicitomas, y a la presencia de características epiteliales en las células del tumor desde un punto de vista ultraestructural ${ }^{3,5,18,23}$. Otros estudios defienden un origen en la glia radial ${ }^{3,5}$, estructura embrionaria precursora de la vía óptica, debido a la preferencia por la localización a nivel hipotálamo-quiasmática ${ }^{5,18}$.

Desde el punto de vista genético y molecular se ha propuesto la posible relación del APM con las proteínas quiméricas con actividad tirosina quinasa que surgen de la translocación de ciertos genes como el BCR del cromosoma 22, tras el análisis citogenético de un caso en el que se observó una translocación 17-22 y que respondió a imanitib, un inhibidor de la actividad tirosina quinasa, tras el fracaso de otras medidas terapéuticas ${ }^{20}$.

El APM puede aparecer en cualquier punto del SNC con un predominio importante de la presentación hipotálamo-quiasmática ${ }^{16}$. La edad media de presentación es de 18 meses, aunque se han descrito casos de aparición en la edad adulta, sin observarse predilección por alguno de los dos sexos. Desde el punto de vista clínico las manifestaciones dependen de la localización de la lesión. Los APM de la zona hipotálamo-quiasmática cursan con alteraciones oculomotoras, alteraciones endocrinas, signos de hipertensión intracraneal (HTIC) por hidrocefalia obstructiva, debilidad y alteraciones del desarrollo. En cuanto a los APM de la fosa posterior se presentan como disfunción cerebelosa o hidrocefalia ${ }^{14,17,18,23}$. Nuestros tres casos debutaron con hidrocefalia, dos de ellos con lesión hipotálamo-quiásmática y otro con lesión en tercio posterior del tercer ventrículo. La edad de presentación de los casos 1 y 3 fue mayor a la edad media descrita. No existe evidencia de que su incidencia sea mayor en los pacientes con neurofibromatosis tipo I, aunque algunos autores comparten la idea de que podría haber sido diagnosticado en muchos casos de forma errónea como astrocitoma de alto grado tras analizar series de paciente con neurofibromatosis tipo I con tumor cerebral ${ }^{12}$.

El APM presenta unos rasgos histo-patológicos característicos: células piloides bipolares monomorfas inmersas en una matriz mixoide abundante, disposición angiocéntrica frecuente y típicamente ausencia de fibras de Rosenthal y cuerpos granulares. Pueden existir figuras mitóticas y se han descrito casos con focos de necrosis y calcifi- caciones. El MIB-1 varía de 2-20\%5,8,9,14,15,17,22,23. A nivel inmunohistoquímico presenta PGFA + de forma difusa, vimentina + , y $\mathrm{S} 100+$. Se describen también casos con sinaptofisina + pero con cromogranina y neurofilamentos negativos ${ }^{3,9,22}$. Nuestros tres casos cumplen criterios histopatológicos de APM destacando la ausencia de fibras de Rosenthal, de cuerpos granulares y la positividad de la PGFA. Hay que añadir que a nivel ultraestructural se han descrito ciertas características epiteliales como la presencia de microvilli superficial, de uno o dos cilios en algunas células y ocasionales uniones e interdigitaciones de membrana. También se ha destacado la presencia de complejos sinápticos y "blebs" citoplasmáticas9. De manera excepcional se ha descrito el sangrado tumoral espontáneo en probable relación a la presencia de teleangiectasias en la pared del componente quístico del $\mathrm{APM}^{10,13}$. En la clasificación actual de la OMS se considera que en AP típicos con limitados cambios pilomixoides no estaría justificado el diagnóstico de $\mathrm{APM}^{22}$.

Respecto al diagnóstico por imagen la RM con gadolinio es la prueba de elección. El APM se caracteriza por aparecer como una lesión bien delimitada, generalmente sólida, isointensa en T1, hiperintensa en T2, que puede realzar de forma homogénea o heterogénea sin una clara predilección, y que se acompaña de discreto edema perilesional y de áreas necrótico-quísticas. Estos hallazgos radiológicos no permiten diferenciar al APM del AP ${ }^{1,4,8,10,12,17,19,21}$. El sangrado tumoral es excepcional aunque parece más frecuente en el APM en comparación con el $\mathrm{AP}^{19}$. En un $15 \%$ de los casos se observa realce meníngeo basal y/o espinal, atribuible a diseminación por $\mathrm{LCR}^{7,14}$. Los hallazgos radiológicos de nuestros tres casos se ajustan a la literatura, encontrando diseminación leptomeníngea en el caso 2. Con RMNespectroscopia se ha descrito disminución de derivados de la colina, creatina y $\mathrm{N}$-acetil-aspartato, a diferencia de los $\mathrm{AP}^{4}$. Otros autores no han encontrado diferencias, aunque se ha reseñado una elevación del índice colina/creatinina a nivel peritumoral que no han presentado los AP y que podría relacionarse con la mayor agresividad del $\mathrm{APM}^{21}$.

Desde el punto de vista pronóstico se ha observado un comportamiento más agresivo del APM respecto del $\mathrm{AP}$, con tendencia a recidivar localmente de forma más frecuente y precoz así como a diseminar por $\mathrm{LCR}^{3,5}$. Las recurrencias del APM tienden a producirse a partir del componente sólido y las del AP a expensas del quístico ${ }^{2}$. La recidiva quística del APM podría sugerir maduración/ diferenciación en $\mathrm{AP}^{4,12}$. Otros autores han puesto en entredicho esta conducta más agresiva basándose en un MIB-1 sin diferencias significativas entre APM y AP y en una serie de 5 casos en los que la evolución del APM fue bastante similar al AP (80\% de supervivencia tras un periodo de 7 11 años de enfermedad $)^{5}$. En un estudio que comparó una serie de 21 APM y de 42 AP de localización hipotálamo- 
Tabla 1

Estudio comparativo entre 21 APM y 42 AP de localización hipotálamo-quiasmática ${ }^{14}$

\begin{tabular}{|l|l|l|}
\hline & APM & AP \\
\hline Periodo medio libre de enfermedad & 26 meses & 147 meses \\
\hline Supervivencia media global & 63 meses & 213 meses \\
\hline Mortalidad (a los 5 años) & $33 \%$ & $17 \%$ \\
\hline
\end{tabular}

Tabla 2

Estudio comparativo entre 18 APM y 13 AP de localización hipotálamo-quiasmática ${ }^{23}$

\begin{tabular}{|l|l|l|}
\hline & APM & AP \\
\hline Pacientes libres de enfermedad (tras un año) & $38^{\prime} 7 \%$ & $69^{\prime} 2 \%$ \\
\hline Supervivencia global (tras dos años) & $38^{\prime} 7 \%$ & $100 \%$ \\
\hline
\end{tabular}

quiasmática se observó un $76 \%$ de recurrencia local en la serie del APM y un $50 \%$ en la del AP, con $15 \%$ de diseminación por LCR en los APM frente a un $0 \%$ los AP (con $\left.\mathrm{p}<0^{\prime} 05\right)^{14}$. En una serie de 90 AP juveniles se observó diseminación en LCR en un $12 \%$ de los casos, y se piensa que un alto porcentaje podría ser atribuible a APM en base a los criterios actuales ${ }^{6}$. Se han descrito periodos libres de enfermedad y supervivencia global menores en APM en dos estudios comparativos con AP (ver tablas 1 y 2$)^{14,23}$. La variante pilomixoide del AP se ha considerado como factor de mal pronóstico ${ }^{8}$. A nivel medular el APM tendría mejor pronóstico que los astrocitomas infiltrantes, pero peor que los $\mathrm{AP}^{15}$.

Respecto al manejo terapéutico del APM no existe consenso en el momento actual. La resección total es el predictor más fiable de un resultado terapéutico favorable $e^{3,17}$. Hay que tener en cuenta que en estos tumores la resección total es difícil, tanto por su tendencia a localizarse en la zona del hipotálamo-quiasmática como por la presencia de una interfase tejido sano vs. tejido patológico poco evidente ${ }^{1}$. Este hecho justifica abordajes quirúrgicos más conservadores con fines diagnósticos como ocurre en nuestros tres casos. La terapia adyuvante se reserva para los casos de recidiva tras la cirugía, resección parcial con crecimiento tumoral demostrado, y en caso de resección parcial en presencia de clínica neurológica, siendo la quimioterapia el tratamiento de elección en pacientes menores de 3-5 años. Por encima de esta edad la combinación de quimioterapia y radioterapia ha demostrado mejores resultados que el uso no combinado de las mismas ${ }^{16}$. Algunas publicaciones destacan que se trata de una entidad con pobre respuesta a la radioterapia y quimioterapia ${ }^{4}$. Se ha descrito respuesta al tratamiento médico con inhibidores de tirosín quinasa en un caso de APM diseminado ${ }^{20}$. En casos de diseminación en neuroeje se ha conseguido mejoría con radioterapia (2 Gy) y temozolamida $(75 \mathrm{mg} / \mathrm{m} 2)^{7}$. En caso de enfermedad localizada en neuroeje la primera opción es la cirugía, teniendo como alternativa la radioterapia y/o quimioterapia y, en caso de no respuesta, la realización de laminectomías descompresivas ${ }^{15}$.

\section{Conclusión}

El APM, de reciente descripción, podría representar un subtipo o variante de AP de comportamiento más agresivo, con más tendencia a la recidiva local y diseminación por LCR, así como con peor pronóstico vital. Se precisan estudios de mayor casuística y seguimiento prolongado para establecer de forma definitiva un consenso terapéutico.

\section{Bibliografía}

1. Arslanoglu, A., Cirak, B., Horska, A., et al.: MR imaging characteristics of pilomyxoid astrocytomas. AJNR Am J Neuroradiol 2003; 24: 1906-1908.

2. Burger, P.C., Cohen, K.J., Rosenblum, M.K., Tihan, T.: Pathology of diencephalic astrocytomas. Pediatr Neurosurg 2000; 32: 214-219.

3. Ceppa, E.P., Bouffet, E., Griebel, R., Robinson, C., Tihan, T.: The pilomyxoid astrocytoma and its relationship to pilocytic astrocytoma: report of a case and a critical review of the entity. J Neurooncol 2007; 81: 191-196.

4. Cirak, B., Horskä, A., Barker, P.B., Burger, P.C., Carson, B.S., Avellino, A.M.: Proton magnetic resonance spectroscopic imaging in pediatric pilomyxoid astrocytoma. Childs Nerv Syst 2005; 21: 404-409.

5. Chikai, K., Onhishi, A., Kato, T., et al.: Clinico-pathological features of pilomyxoid astrocytoma of the optic pathway. 
Acta Neuropathol 2004; 108: 109-114.

6. Darwish, B., Koleda, C., Lau, H., Balakrishnan, V., Wickremesekera, A.: Juvenile pilocytic astrocytoma "pilomyxoid variant" with spinal metastases. J Clin Neurosci 2004; 11 : 640-642.

7. Enting, R.H., van der Graaf, W.T., Kros, J.M., Heesters, M., Metzemaekers, J., den Dunnen, W.: Radiotherapy plus concomitant and adjuvant temozolomide for leptomeningeal pilomyxoid astrocytoma: a case study. J Neurooncol 2006; 80: 107-108.

8. Fernandez, C., Figarella-Branger, D., Girard, N., et al.: Pilocytic astrocytomas in children: prognostic factors----a retrospective study of 80 cases. Neurosurgery 2003; 53: 544553.

9. Fuller, C.E., Frankel, B., Smith, M., et al.: Suprasellar monomorphus pilomyxoid neoplasm: an ultastructural analysis. Clin Neuropathol 2001; 20: 256-262.

10. Gottfried, O.N., Fults, D.W., Townsend, J.J., Couldwell, W.T.: Spontaneos hemorrhage associated with a pilomyxoid astrocytoma. J Neurosurg 2003; 99: 416-420.

11. Jänisch, W., Schreiber, D., Martin, H., Gerlach, H.: Diencephalic pilocytic astrocytoma with clinical onset in infancy. Biological behaviour and pathomorphological findings in 11 children. Zentralbl Allg Pathol 1985; 130: 31-43.

12. Khanani, M.F., Hawkins, C., Shroff, M., et al.: Pilomyxoid astrocytoma in a patient with neurofibromatosis. Pediatr Blood Cancer 2006; 46: 377-380.

13. Komakula, S.T., Fenton, L.Z., Kleinschmidt-DeMasters, B.K., Foreman, N.K.: Pilomyxoid astrocytoma: neuroimaging with clinicopathologic correlates in 4 cases followed over time. J Pediatr Hematol Oncol 2007; 29: 465-470.

14. Komotar, R.J., Burger, P.C., Brem, H., et al.: Pilocytic and pilomyxoid hypothalamic/chiasmatic astrocytomas. Neurosurgery 2004; 54: 72-79.

15. Komotar, R.J., Carson, B.S., Rao, C., Chaffee, S., Goldthwaite, P.T., Tihan, T.: Pilomyxoid astrocytoma of the spinal cord: report of three cases. Neurosurgery 2005; 56: 206-210.

\section{Comentario al trabajo Astrocitomas pilomixoides. Pre- sentación de tres casos y revisión de la literatura de $\mathrm{M}$. Domínguez Páez y cols}

En este artículo se presentan tres nuevos casos de una patología de reciente descripción y con la que muchos no estábamos familiarizados hasta hace poco tiempo. La principal aportación de este trabajo es el sumar datos acerca de una neoplasia cuyo espectro clínico, radiológico e histológico se está aún definiendo. En la anterior clasificación de los tumores del sistema nervioso de la OMS se reconocía la existencia de astrocitomas pilocíticos de localización predominantemente hipotalámica-quiasmática que mostraban mayor tendencia a la diseminación por
16. Komotar, R.J., Mocco, J, Carson, B.S., et al.: Pilomyxoid astrocytoma: a review. MedGenMed 2004; 6: 42.

17. Komotar, R.J., Mocco, J., Jones, J.E., et al.: Pilomyxoid astrocytoma: diagnosis, prognosis, and management. Neurosurg Focus 2005; 18: E7.

18. Komotar, R.J., Mocco, J., Zacharia, B.E., et al.: Astrocytoma with a pilomyxoid features presenting in an adult. Neuropathology 2006; 26: 89-93.

19. Linscott, L.L., Osborn, A.G., Blaser, S., et al.: Pilomyxoid astrocytoma: expanding the imaging spectrum. Am J Neurorradiol 2008; 29: 1861-1866.

20. Meléndez, B., Fiaño, C., Ruano, Y., Hernández-Moneo, J.L., Mollejo, M., Martínez, P.: BCR gene disruption in a pilomyxoid astrocytoma. Neuropathology 2006; 26: 442-446.

21. Morales, H., Kwock, L., Castillo, M.: Magnetic resonance imaging and spectroscopy of pilomyxoid astrocytomas: case reports and comparison with pilocytic astrocytomas. J Comput Assist Tomogr 2007; 31: 682-687.

22. Sheithauer, B.W., Hawkins, C., Tihan, T., et al.: Pilocytic astrocytoma. En: Louis, D.N., Ohgaki, H., Wiestler, O.D., et al, eds.: World Health Organization classification of tumours. Pathology and genetics of tumours of the nervous system. 2007: 14-21.

23. Tihan, T., Fisher, P.G., Kepner, J.L., et al.: Pediatric astrocytomas with monomosphous pilomyxoid features and a less favorable outcome. J Neuropathol Exp Neurol 1999; 58: 1061-1068.

Domínguez-Páez, M.; Weil-Lara, B.; Rodríguez-Barceló, S.; Medina-Imbroda, J.M.; Puch-Ramírez, M.; Ros-López, B.; Arráez-Sánchez, M.A.: Astrocitomas pilomixoides. Presentación de tres casos y revisión de la literatura. Neurocirugía 2010; 21: 22-29.

Correspondencia: Servicio de Neurocirugía. Hospital Regional Universitario Carlos Haya. Avda Carlos Haya, s/n. 29010. Málaga. E- mail: lenin_@hotmail.com

el líquido cefalorraquídeo (LCR) y un comportamiento atípico/agresivo ${ }^{1}$. Son estas las características clínicas y topográficas típicas de la nueva entidad reconocida en la nueva edición de esta clasificación como astrocitoma pilomixoide 5 . Sin embargo, se ha de tener en cuenta que en la nueva clasificación de la OMS se sigue reconociendo la posibilidad de la siembra por LCR de astrocitomas pilocíticos típicos, también predominantemente hipotalámicos (en tanto que es la localización más frecuente de los astrocitomas pilomixoides, es la segunda más frecuente 
en los astrocitomas pilocíticos, que por otro lado son tumores de mayor incidencia). Ambas entidades son casi indistinguibles en cuanto a su presentación clínica y radiológica por lo que el diagnóstico se fundamenta en los hallazgos histológicos que se han comentado en el artículo. Se ha descrito que los astrocitomas pilomixoides se presentan a una edad más temprana, tienen una ligera mayor tendencia a mostrar signos de sangrado o de diseminación en los estudios de imagen iniciales, y que presentan un margen peor definido en los estudios de RM espectroscopia y en la cirugía ${ }^{4}$, pero la diferencia fundamental radica en su comportamiento biológico, con una mayor tendencia a la recidiva y diseminación que los astrocitomas pilocíticos ${ }^{2,3,5}$. Un mayor número de casos, y sobre todo los estudios de biología molecular, permitirán dilucidar si nos encontramos ante dos entidades diferentes o los extremos del espectro de una misma entidad, pero el diagnóstico de un astrocitoma pilomixoide ha de hacer prever un peor pronóstico e indicar un manejo terapéutico más agresivo².

El tratamiento quirúrgico es el primer paso en el tratamiento de estas lesiones, siendo la resección completa el objetivo del mismo. Sin embargo, dada la frecuente localización en torno al área hipotalámica este tratamiento no es posible en ocasiones. Una vez obtenido el diagnóstico se ha de realizar una RM de todo el neuroeje dada la tendencia a la diseminación de este tumor. No queda claro en qué momento se ha de indicar el tratamiento oncológico con radio o quimioterapia, aunque puede emplearse en recaídas tras resección completa, en los casos sintomáticos en que el tumor no haya podido ser resecado y en las resecciones parciales cuando se observe progresión del resto tumoral ${ }^{3}$. En este sentido el trabajo anterior muestra cómo al menos dos de los casos experimentaron una respuesta positiva a la radioterapia o quimioterapia.

Será importante seguir analizando los casos de más pacientes con astrocitoma pilomixoide, interpretando con cautela aquéllos que se salgan de las características típicas de edad, localización, histología y comportamiento ${ }^{2}$, particularmente cuando el diagnóstico se obtenga de muestras de biopsia (parciales por definición). Debemos tener en mente el hecho de que determinados astrocitomas pilocíticos pueden mostrar áreas histológicamente similares a los astrocitomas pilomixoides ${ }^{2}$, sin que esto cambie su diagnóstico, que condicionará la información pronóstica y en ocasiones la indicación o no de tratamientos radio- o quimioterápicos.

\section{Bibliografía}

1. Burger, P.C., Scheitauer, B.W., Paulus, W., Szymas, J., Giannini, C., Kleihues, P.: Pilocytic Astrocytoma. En Kleihues, P., Cavenee, W.K. (ed). Pathology and Genetics of Tumors of the Nervous System. Lyon; IARC Press, 2000; pp.45-51.

2. Ceppa, E.P., Bouffet, E., Griebel, R., Robinson, C., Tihan, T.: The pilomyxoid astrocytoma and its relationship to pilocytic astrocytoma: report of a case and a critical review of the entity. J Neurooncol 2007; 81: 191-196.

3. Komotar, R.J., Mocco, J., Jones, J.E. et al.: Pilomyxoid astrocytoma: diagnosis, prognosis, and management. Neurosurg Focus [Revista electrónica] 2005; 18: Artículo 7 [4p]. Disponible en: http://thejns.org/doi/pdf/10.3171/foc.2005.18.6.8

4. Linscott, L.L., Osborn, A.G., Blaser, S. et al.: Pilomyxoid astrocytoma: expanding the imaging spectrum. AJNR Am J Neuroradiol 2008; 29: 1861-1866.

5. Scheitauer, B.W., Hawkins, C., Tihan, T., VandenBerg, S.R., Burger, P.C.: Pilocytic Astrocytoma. En Louis, D.N., Ohgaki, H., Wiestler, O.D., Cavenee, W.K. (ed). WHO Clasification of Tumors of the Central Nervous System. Lyon; IARC press, 2007; pp.14-21.

A. Pérez-Núñez Madrid 